\title{
STATIC PLANE-SYMMETRIC SOLUTION OF A SCALAR-TENSOR THEORY OF GRAVITATION
}

\author{
D. R. K. REDDY and V. U. M. RAO'
}

(Received 1 December 1980; revised 13 April 1982)

\begin{abstract}
Vacuum field equations in a scalar-tensor theory of gravitation, proposed by Ross, are obtained with the aid of a static plane-symmetric metric. A closed form exact solution to the field equations in this theory is presented which can be considered as an analogue of Taub's empty space-time in Einstein's theory.
\end{abstract}

\section{Introduction}

Brans and Dicke [1] have formulated a scalar-tensor theory of gravitation in which the tensor field alone is geometrised and the scalar field is alien to geometry. Later, Sen and Dunn [7] proposed a scalar-tensor theory of gravitation in a modified Riemannian manifold in which both the scalar and tensor fields have intrinsic geometrical significance. Recently Ross [5] has constructed a new scalar-tensor theory of gravitation using the Weyl formulation of Riemannian geometry. The scalar field in this theory is given an important geometrical role to play and is related to the integrable change in length of a vector as it is transported from point to point in space-time. It is pointed out that this theory is an alternative to the usual Brans-Dicke [1] formalism in regions of space free of mass and charge densities. The scalar field enters the two theories very differently.

The field equations given by Ross [5] for the combined scalar and tensor fields in the regions of space free of mass and charge densities are

\footnotetext{
'Department of Applied Mathematics, Andhra University, Waltair-530 003, India.

(C) Copyright Australian Mathematical Society 1983
} 


$$
\begin{aligned}
& S_{i j}-\frac{1}{2} g_{t j} S=0 \text {, and } \\
& \phi_{\| k}^{k k}=g^{k j} \phi_{|k| j}-g^{k j} \phi_{\mid \imath}\{k j, i\}-4 \phi_{\mid k} \phi^{\mid k}=0 \text {, where } \\
& \left.S_{i j}=R_{i j}-2 \phi_{|,|}-2 \phi_{\mid \imath} \phi_{\mid j}+2 g_{i j} \phi^{\mid k} \phi_{\mid k}-g_{\imath,} g^{i k} \phi_{\mid \eta k}\right\} \\
& +2 \phi_{\mid k}\{i j, k\}+g_{i j} g^{l k}\{l k, m\} \text {, and } \\
& \boldsymbol{\phi}^{k} \equiv g^{k} \boldsymbol{\phi}_{i}
\end{aligned}
$$

A double vertical bar here denotes covariant differentiation and $\phi$ is the fundamental scalar field in the theory. Further, $R_{i j}$ is the usual contracted Riemann curvature tensor and $\{i j, k\}$ is the Christoffel symbol of the second kind. By using a static spherically symmetric solution to the field equations, Ross [5] has also shown that the present theory agrees with the Brans-Dicke formalism, in the experimental results. Krori and Nandy [3] have shown that an analogue of Birkhoff's theorem of general relativity is valid in this theory when the scalar field is time independent. The problem of scalar-tensor fields has been studied very little so far in the plane symmetric space-time of Taub [8]. Recently Reddy [4] has obtained static plane-symmetric solutions in Brans-Dicke and Sen-Dunn scalar tensor theories of gravitation. This theory of gravitation has not been studied so far in the plane-symmetric space-time. We have taken up the investigation of the field equations of this scalar-tensor theory in the plane-symmetric space-time of Taub [8] and have obtained an exact static solution which is a true generalisation of Taub's empty space-time static solution to the case when the scalar field is present. Also, since exact solutions of these field equations are scarce, it is worthwhile to obtain analytic solution of a problem with more than one field coupled nonlinearly.

\section{Field equations and solutions}

We consider the static plane symmetric line element

$$
d s^{2}=e^{2 \alpha}\left(d t^{2}-d x^{2}\right)-e^{2 \beta}\left(d y^{2}+d z^{2}\right)
$$

where $\alpha$ and $\beta$ are functions of $x$ only. The plane symmetry assumed implies that the scalar field $\phi$ shares the same symmetry.

Taking $\phi$ as a function of $x$ only the Ross vacuum field equations (1), in the plane symmetric space-time (2), can be written as

$$
\left.\begin{array}{l}
\beta^{\prime 2}+2 \alpha^{\prime} \beta^{\prime}+3 \phi^{\prime 2}-2 \phi^{\prime}\left(\alpha^{\prime}+2 / \beta^{\prime}\right)=0 \\
\alpha^{\prime \prime}+\beta^{\prime \prime}+\beta^{\prime 2}-2 \phi^{\prime \prime}+\phi^{\prime 2}-2 \phi^{\prime} \beta^{\prime}=0 \\
2 \beta^{\prime \prime}+3 \beta^{\prime 2}-2 \alpha^{\prime} \beta^{\prime}-2 \phi^{\prime \prime}+\phi^{\prime 2}+2 \phi^{\prime}\left(\alpha^{\prime}-2 \beta^{\prime}\right)=0 \\
\phi^{\prime \prime}+2 \beta^{\prime} \phi^{\prime}-4 \phi^{\prime 2}=0
\end{array}\right\}
$$

Here a superscript prime indicates differentiation with respect to $x$. 
It can be easily verified that, when the scalar field is constant, the field equations (3) yield a solution which describes an empty space-time discussed by Taub [8] in Einstein's theory.

When $\phi$ is a function of $x$ only, the static non-flat plane symmetric vacuum solution of Ross field equations (3) can be written as

$$
d s^{2}=e^{2 \phi} d s_{\text {Einsten }}^{2}
$$

where

$$
e^{-2 \phi}=-A \log |x|
$$

Here $A$ is a constant and

$$
d s_{\text {Enstein }}^{2}=x^{-\frac{1}{2}}\left(d t^{2}-d x^{2}\right)-x\left(d y^{2}+d z^{2}\right)
$$

is the static non-flat plane symmetric solution due to Taub [8] in Einstein's theory.

\section{Discussion}

In pursuing the study of scalar-tensor theories of gravitation it has been a common practice to compare the results of these theories with those of Einstein's theory under similar conditions to find out the influence of the scalar field $\phi$. With this objective we have taken up this investigation.

Ross [6] has shown that the vacuum field equations of his theory are conformally equivalent in form to the Einstein field equations in empty space. Ross [5] has also pointed out that the Weyl gauge transformation,

$$
\bar{g}_{\imath \lambda}=f\left(x^{l}\right) g_{\imath \jmath}
$$

where $f\left(x^{l}\right)$ is an arbitrary scalar field used in his theory, which is very similar to the conformal transformation discussed by Dicke [2]. Hence it is obvious that the vacuum solutions of Ross theory would be conformally related to the vacuum solutions of Einstein theory. But the point of interest in finding exact vacuum solutions of Ross theory is mainly to see what form the fundamental scalar field has. Then all vacuum solutions of Ross theory can be found by multiplying the vacuum solutions of Einstein's theory by appropriate conformal factors. It can be seen that our solution (4) constitutes an example of this situation in Ross theory.

From (4) and (5) it is interesting to note that the plane symmetric vacuum metric in Ross scalar-tensor theory is conformally related to the Einstein vacuum metric. This conformal relationship occurs in this theory because of the fact that the Ross vacuum equations and Einstein vacuum equations are conformally equivalent in form (see [6]). Also this conformal relationship always does occur in 
vacuum because the present theory holds in regions of space which have no charge and mass densities. It may be mentioned in this connection that the scalar field $\phi$ in Ross theory plays a rather elegant geometrical role. Also this solution is due to an infinite plane parallel to the $(y, z)$ plane.

It is well known that Taub's empty space-time (5) has an intrinsic singularity at $x=0$ and hence the plane symmetric space-time in Ross theory given by (4) also possesses an intrinsic singularity at $x=0$. Thus we see that there is one-to-one correspondence between singularities of the Einstein vacuum solution and the vacuum solution of Ross theory of gravitation.

In conclusion, we hope that the above investigation may give some clue to a deeper understanding of Ross scalar-tensor theory of gravitation.

\section{Acknowledgements}

The authors are grateful to the referee for his constructive comments towards the improvement of this paper. The second author (V. U. M. Rao) is also grateful to the CSIR, India, for a fellowship.

\section{References}

[1] C. Brans and R. H. Dicke, "Mach's principle and a relativistic theory of gravitation", Phys. Rev. 124 (1961), 925-935.

[2] R. H. Dicke, "Mach's principle and invariance under transformation of units", Phys. Rev. 125 (1962), 2163-2167.

[3] K. D. Krori and D. Nandy, "Birkhoff's theorem and scalar-tensor theories of gravitation", $J$. Phys. A. Math. Nucl. Gen. 10 (1977), 993-996.

[4] D. R. K. Reddy, "Static plane-symmetric solutions in Brans-Dicke and Sen-Dunn theories of gravitation", J. Phys. A. Math. Gen. 10 (1977), 55-58.

[5] D. K. Ross, "Scalar-tensor theory of gravitation", Phys. Rev. D 5 (1972), 284-290.

[6] D. K. Ross, "Lagrangian formulation of a geometrical scalar-tensor theory of gravitation", Gen. Relativity Gravitation 6 (1975), 157-164.

[7] D. K. Sen and K. A. Dunn, "A scalar-tensor theory of gravitation in a modified Riemannan manifold", J. Math. Phys. 12 (1971), 578-586.

[8] A. H. Taub, "Empty space-times admitting a three parameter group of motions", Ann. Math. 53 (1951), 472-490. 